\title{
GANADERÍA Y CAMBIO CLIMÁTICO: UNA INFLUENCIA RECÍPROCA
}

\author{
Alberto Lorente Saiz. Estudiante de la Licenciatura de Geografía. Universidad de \\ Alicante (España) \\ Correo electrónico: albertolorentesaiz@gmail.com
}

Recibido: 2 de diciembre de 2010. Aceptado: 23 de diciembre de 2010.

\section{RESUMEN}

La preocupación por el deterioro ambiental y por el cambio climático son temas que están muy de actualidad. Esta reflexión aproxima al lector a las interrelaciones que se establecen entre la producción ganadera y el medio físico. La situación de la ganadería actual viene dada por el sistema económico-productivo actual. Un sistema básicamente injusto e insostenible que rige las actividades humanas a escala mundial. En este sentido, tanto el deterioro ambiental como el actual calentamiento global son una de las consecuencias negativas del desarrollo de estas actividades humanas, que están llevando al planeta a su límite. En estas consecuencias ambientales, el sector agropecuario, relacionado con los procesos de elaboración, distribución y consumo, tiene un papel principal en el que contribuye, a la vez que se ve perjudicado, tanto directa como indirectamente.

Palabras clave: Ganadería, Ambiente, Cambio climático, Capitalismo.

\begin{abstract}
The worry for the environmental deterioration and for the climate change they are topics that are very of current importance. This reflection brings the reader near to the interrelationships that are established between the cattle production and the environment. The situation of the current ranching comes given by the economic productive current system. A basically unjust and untenable system that governs the human activities on a worldwide scale. Both the environmental deterioration and the current global warming are one of the negative consequences of the development of these human activities, which are leading to the planet to his limit. In these environmental consequences, the agricultural sector, related to the processes of production, distribution and consumption, has a principal paper in the one that contributes, simultaneously that meets harmed, so much direct as indirectly.
\end{abstract}

Key words: Livestock, Environment, Climatic Change, Capitalism. 


\title{
INTRODUCCIÓN
}

\author{
Ni la sociedad, ni el hombre, ni ninguna otra cosa \\ deben sobrepasar para ser buenos \\ los límites establecidos por la naturaleza \\ Hipócrates (s. V a.C. - s. IV a.C.), médico griego
}

La tierra es un ente dinámico compuesto por millones de elementos diferentes que se encuentran interrelacionados entre sí. Estos elementos dependen unos de otros y a partir de su relación se desarrollan todos los fenómenos del planeta. Por lo tanto el medio esta en constante transformación, se renueva y desarrolla sin cesar, y siempre hay algo que nace y evoluciona y algo que muere y caduca (Politzer, 1985).

El cambio climático sería un fenómeno más, resultado de la relación de los diferentes agentes del territorio. Es cierto que, a día de hoy, aún no se conocen a ciencia cierta sus causas ni consecuencias, aunque también es verad que sí existe una opinión muy generalizada que defiende que el cambio climático se debe a causas antropogénicas. Esta teoría, basada en los intercambios de energía calorífica sol-tierra, recibe el nombre de «calentamiento global por efecto invernadero» ${ }^{1}$ y esta apoyada por organismos supranacionales como la ONU. No obstante, es necesario destacar que también existe un reducido sector de científicos que niegan estas ideas ${ }^{2}$. A pesar de ello, este artículo respaldará la teoría mayoritaria. En la teoría del cambio climático como consecuencia del «efecto invernadero», tienen un papel fundamental los denominados «Gases de Efecto Invernadero» (en adelante GEI) ${ }^{3}$, los cuales, según la teoría mayoritaria, han aumentado en proporción debido a las actividades humanas. Este tipo de gases absorben una porción de la radiación que emite la tierra al exterior, por lo que al aumentar su concentración, la tierra libera menos calor y la temperatura del planeta aumenta (Barros, 2005). Esto supone un problema muy alarmante ya que, debido al carácter dialéctico de los elementos del territorio, una modificación en las pautas atmosféricas no solo afectará al sistema climático, si no que tendrá consecuencias en todo el conjunto del espacio geográfico.

\footnotetext{
${ }^{1}$ Esta teoría consiste en que el sol emite energía electromagnética a la tierra, mientras que la tierra, como consecuencia de su calentamiento, expulsa parte de esta energía al espacio exterior. La atmósfera resulta transparente a los rayos emitidos por el sol, mientras que resulta, en cierta medida, opaca a la energía emitida por la tierra al exterior. Esta propiedad la tienen también otros materiales como pueden ser el cristal o el plástico, que son los materiales que se usan para construir los invernaderos. La tierra tan solo libera energía en una determinada longitud de onda denominada "ventana de radiación". Esta propiedad de la atmósfera es la que hace que parezca un invernadero, y sin ella las temperaturas en la tierra serían mucho menores.

2 Algunos expertos defienden que el cambio climático actual se debe a factores naturales imposibles de analizar con la tecnología actual, como puede ser el grado de inclinación del sol o a la influencia de otros factores siderales. Estos expertos, autodenominados "herejes" (por no seguir a la mayoría), opinan que la teoría que defiende las causas antropogénicas del cambio climático esconde detrás una manipulación de índole marxista.

${ }^{3}$ Los principales gases de efecto invernadero son, por orden de importancia, el vapor de agua, el dióxido de carbono $\mathrm{CO}^{2}$, el metano $\mathrm{CH}^{4}$ y el óxido nitroso $\mathrm{N}^{2} \mathrm{O}$, además de tres gases industriales fluorados: hidrofluorocarbonos (HFC), perfluorocarbonos (PFC) y hexafluoruro de azufre $\left(\mathrm{SF}^{6}\right)$. Los cuatro GEI principales permanecen en la atmósfera de manera natural; sin embargo, la acción del hombre ha modificado sus porcentajes en las últimas décadas.
} 
Como ya se ha mencionado, a día de hoy no se puede hablar con exactitud de las consecuencias de este fenómeno, sin embargo, el consenso científico actual dice que en las próximas décadas habrá un aumento de $2^{\circ} \mathrm{C}$ en las temperaturas medias y (si no se cambia la dinámica humana actual) de $8^{\circ} \mathrm{C}$ hacia el año 2100 (Grain, 2009). Estos cambios en el factor temperatura, según los informes 2001 y 2007 del IPCC $^{4}$ sobre el cambio climático, ya se están haciendo notar en diferentes sistemas físicos y humanos, como por ejemplo en el deshielo del permafrost, la contracción de los glaciares, el aumento del nivel del mar o, en relación con el tema a tratar, el desplazamiento en el tiempo de las labores agrosilvopastoriles. Esto tan solo supone el principio de lo que, según los expertos, serán unas consecuencias que provocarán cambios radicales tanto en el medio como en los modos de vida de los seres humanos, sobre todo aquellos que dependen directamente de la naturaleza como es el sector agropecuario.

Siguiendo esta teoría, es posible afirmar que el cambio climático se debe a causas antropogénicas, y en este sentido, la ganadería como actividad humana, contribuye de manera muy relevante. En relación con ello, el sector agropecuario, al igual que la mayoría de las actividades humanas, viene determinado por el sistema económico actual. Este sistema no es otro que el clásico «capitalismo», hoy día llamado «neoliberalismo» (Segrelles, 2004). Desde hace varios siglos (Bidwai, 2007), las actividades humanas se rigen según la lógica de este sistema, hoy día controlado por un grupo reducido de grandes empresas transnacionales. Éstas buscan el máximo beneficio a toda costa, considerando todos los elementos del territorio como recursos explotables ilimitados. La ley de la oferta y la demanda, el "libre" mercado y la libre competencia, el fordismo, la homogeneización de los productos y de los deseos de los consumidores, el objetivo único de conseguir el máximo beneficio y la máxima acumulación de capital, son algunas de sus características esenciales. Además de esto, es un modelo económico que, a escalas mundial y local, ha traído consecuencias negativas como la polarización de la riqueza, el desequilibrio entre territorios, y que además está conduciendo a la destrucción del planeta.

En relación con el asunto que aborda el artículo, la lógica neoliberal se sustenta en una estrecha relación consumo-producción ${ }^{5}$ que ha provocado cambios en los modelos de producción agropecuaria, así como en el consumo de las diferentes sociedades, colaborando de esta manera en la degradación ambiental y en la crisis climática. En definitiva, una crisis natural que en opinión de diferentes autores como E. Leff, citado por J. A. Segrelles (2008), esconde detrás una auténtica “crisis de civilización".

\footnotetext{
${ }^{4}$ Grupo Intergubernamental de Expertos sobre el cambio climático. PNUMA.

5 Por un lado aparece una rama consumidora, que sería toda la población que tiene suficiente poder económico para poder adquirir bienes y servicios que cubran otras necesidades además de las primarias. Esta rama consumidora es la que sustenta la otra rama, que sería la producción. Por lo que debe ser controlada, a toda costa, mediante la publicidad y los medios de comunicación, creando una sociedad consumista manipulada. El otro eje principal es, como ya se ha indicado, la producción y la distribución. Ésta se encarga de crear los bienes que posteriormente serán consumidos por las sociedades y que son fabricados para satisfacer las necesidades (reales o creadas) de la demanda.
} 


\section{GANADERÍA Y AMBIENTE}

Los primeros usos ganaderos se establecieron respetando un equilibrio entre el ser humano, el ganado y el medio donde se realizaba su explotación. Esto fue así hasta la intrusión de la lógica capitalista en las formas de producir y de consumo. Hoy en día cada vez quedan menos explotaciones que no se guíen por esta lógica, es decir, que no busquen obtener un máximo beneficio económico, considerando la naturaleza y los animales como simples materias primas explotables para obtener la mayor rentabilidad y la máxima acumulación de capital.

La representación de las ideas capitalistas en la producción agrícola se encarnan en el modelo de producción industrial o intensiva. El modo de producción que lleva asociado, pretende conseguir un producto homogéneo que cumpla con las exigencias del mercado, tanto en características como en cantidad. Para conseguir sus objetivos, el modo de producción intensivo se basa en el empleo de técnicas modernas como la selección genética, la explotación intensiva y el empleo de productos químicos para mejorar la producción. La apuesta por este tipo de explotación provocó, en la década de los años sesenta, la conocida como revolución verde ${ }^{6}$, que aplicada en la ganadería se conoció como revolución ganadera. Este fenómeno trajo consigo un aumento extraordinario de la producción de alimentos, y junto a él se forjaron unas esperanzas muy optimistas en lo que podía representar el final del problema del hambre en el mundo. Sin embargo, pese a que se consiguieron objetivos como la competitividad, la adaptación al mercado mediante precios bajos o el enriquecimiento de las empresas transnacionales que invirtieron en este tipo de producción agraria, no se consiguió el principal cometido: eliminar el hambre. En relación con esto, muchos expertos están de acuerdo en que "la producción de alimentos para satisfacer a una población mundial en crecimiento llevó en las últimas décadas a una agricultura y ganadería a gran escala, sin control y sin visión de las consecuencias negativas sobre el ambiente" (Gaudín, 2010).

Antes de profundizar en los diferentes tipos de ganadería, es conveniente explicar como influye la gran distribución en el desarrollo del sector agropecuario. Como ya se ha indicado arriba, las grandes firmas transnacionales son las que establecen y siguen el binomio consumo-producción característico de la lógica neoliberal. Son empresas que tienen un gran poder para influir y determinar tanto el consumo como la producción. Cabe destacar que dicho poder surgió a partir de la década de los años ochenta mediante la concentración e internacionalización de sus actividades productivas y comerciales. En efecto, este proceso se desarrolló como consecuencia de la competencia directa que existe entre grandes cadenas distribuidoras como Wal-Mart o Carrefour ${ }^{7}$. A partir de dichas estrategias competitivas, éstas y otras grandes entidades han formado un oligopolio, auspiciado por unos consumidores que, conducidos por una publicidad atrayente, se abastecen en sus hipermercados, supermercados, supermercados de descuento, etc. En este sentido, es posible deducir que, al controlar la oferta y la demanda, controlan también los patrones de producción. Como ejemplo ilustrativo de esta idea, según los datos de Alimarket (2005) utilizados por J. A. Segrelles (2010), la

\footnotetext{
${ }^{6}$ Así se conoce al extraordinario crecimiento de la producción agrícola entre las décadas de los años sesenta y ochenta, período en el que se consiguió un aumento del $250 \%$ en la producción mundial de grano. ${ }^{7}$ Wal Mart, por ejemplo, facturó 378.000 millones de dólares en el año 2007, lo que equivale al 2\% del
PIB de Estados Unidos y a una tercera parte del de España (Segrelles, 2010).
} 
cadena alimentaria europea quedaría representada de la siguiente manera: 3,2 millones de productores se relacionan con 160 millones de consumidores, con la intermediación y control de 110 centrales, grupos de compra y grandes cadenas de distribución. Queda así reflejada la magnitud del poder que tiene la gran distribución.

Desde el plano agropecuario, este sector se encarga de satisfacer las necesidades productivas de la distribución, tanto en tiempo como en cantidad. Al concentrarse la oferta en muy pocas centrales, y todas ellas con unos esquemas y objetivos comunes (precios bajos, máxima rentabilidad en el menor tiempo), el producto que se elabora y el producto que se oferta serán muy similares. La estrategia principal de la gran distribución es reducir los costes para así poder obtener un artículo que se pueda vender a bajo precio, a la vez que se le pueda sacar la máxima rentabilidad. Para ello se suele recurrir a una deslocalización de la cadena productiva, es decir, se localizan en regiones o países donde los costes de producción son menores. Además, otra consecuencia que subyace en esa búsqueda basada en la obtención de un producto homogéneo y que satisfaga las necesidades de un mercado imprevisible, es la necesidad de obtener un excedente cárnico. Este stock en muchos casos se pierde al no reunir las características apropiadas para su puesta en el mercado, mientras que en otras ocasiones se deshecha simplemente por no haberse vendido. Por lo tanto, se observa cómo en un mercado capitalista solo es consumida una pequeña parte del alimento producido. Esto resulta desequilibrado y completamente injusto, sobre todo teniendo en cuenta los millones de personas que padecen desnutrición en el mundo, además de los importantes daños ambientales que provoca esa acción.

En definitiva, en relación con los sistemas de producción agropecuaria, la gran distribución, para poder conseguir sus objetivos y poder contar con la cantidad necesaria de productos y a tiempo, pretende imponer un modelo basado en pocos productores, de gran tamaño y con una capacidad técnica y tecnológica que le de la posibilidad de controlar la cantidad, el aspecto y la temporalidad del producto ofertado (Segrelles, 2010). En este sentido, no todos los productores son aptos para ese modelo productivo, ni tampoco las condiciones naturales del medio pueden soportar los sistemas de producción impuestos por el mercado.

\subsection{Tipos de explotación}

En este contexto, cabe diferenciar entre la explotación tradicional o extensiva y la de tipo industrial o intensiva. Ambas tienen una influencia desigual en el espacio geográfico, entendido éste de la misma forma en que se ha expuesto en la introducción.

Por un lado se encuentra la explotación tradicional o extensiva, que en la actualidad también se le llama familiar por oposición al modelo industrial y capitalizado. Este tipo de explotación se ha dado desde los primeros aprovechamientos ganaderos de la historia. En ellos se buscaba la adaptación interrelacionada entre el animal, la explotación humana y el medio físico, llegando a un equilibrio en el que se daba un uso sostenible de los recursos y unos aprovechamientos sometidos a los ciclos naturales. El fruto del modelo tradicional son los denominados agroecosistemas (unión entre sistema biológico y sistema agrario). Sin embargo, este tipo de explotación es menos rentable económicamente que la explotación intensiva, entre otros motivos porque utiliza más superficie de terreno para criar menos cantidad de animales, y porque el producto que 
genera no siempre se adapta al patrón del mercado, ni en características, ni en cantidad, ni en tiempo. En cualquier caso, se trata de un producto más saludable y más respetuoso con el ambiente, ya que en muchas ocasiones la adaptación a esos patrones mercantiles (sobre todo el de los costes mínimos) desencadena un empeoramiento de la calidad de los productos obtenidos. En otro sentido, si se tienen en cuenta unos esquemas más humanos, el modelo tradicional representa el medio de subsistencia de cientos de millones de familias en el planeta, sobre todo en las regiones empobrecidas.

Bajo una óptica ambiental, el modelo ganadero familiar contribuye a la degradación del territorio pero en una proporción bastante inferior a la contribución de los sistemas productivos industriales. Cabe destacar que en la explotación extensiva, el aspecto que más repercusión ambiental tiene es el desmonte para la obtención de pastos. Por otro lado, también puede influir en la degradación de las áreas de pastizales como consecuencia de la sobreexplotación (sobre todo en las regiones áridas o semiáridas donde los cultivos tardan más en desarrollarse). Ahora bien, debido a su distribución de manera extensiva en el territorio, se podría decir que su impacto negativo en cuanto a la emisión de flujos contaminantes o en la compactación del suelo es menor que el modelo de producción intensivo. Esto se debe a que al encontrarse repartido en la superficie no se supera la capacidad de carga del suelo. Es más, en la mayoría de los casos, más que contaminar supone una carga de nutrientes y una contribución al desarrollo de esa superficie. Por lo tanto, en el lado opuesto, es preciso resaltar también los aspectos positivos que tiene este tipo de explotación en el medio. Según se ha indicado, forma un estrecho vínculo con el medio. En esta línea, cabe destacar que aporta de manera sostenible abono natural, control de vegetación arbustiva y de la biomasa combustible en zonas forestales, además de contribuir a la preservación de la biodiversidad. En conclusión, el modelo tradicional o familiar es el tipo de explotación que menos interviene en la degradación del ambiente, pero al depender de los ciclos naturales es el que más se ve perjudicado por los cambios que se producen en el medio físico.

Por otro lado, el modelo intensivo o industrial es minoritario en cuanto a extensión pero ofrece unas cifras de producción y unas repercusiones, tanto en el medio como en toda la cadena agroalimentaria, que le conceden un valor capital. El ganado se encuentra estabulado bajo condiciones de humedad, luz y temperatura creadas de manera artificial. Además, las especies son seleccionadas y modificadas genéticamente para obtener una mayor productividad. Es decir, la producción se halla adaptada a las exigencias del mercado y es mucho más rentable que el modelo extensivo debido a la explotación intensiva del territorio. Sin embargo, el consumo de recursos es desproporcionado al tener que crear, por ejemplo, atmósferas artificiales.

Siguiendo el esquema anterior, el modelo de producción intensiva genera poco empleo, al mismo tiempo que los beneficios no van destinados a mejorar la calidad de vida de los trabajadores. En este sentido, cabe mencionar que en muchas ocasiones, debido a la política de bajo coste, los productores perciben unos beneficios muy escasos. Posteriormente, el mismo producto es vendido por la gran distribución a unos precios muy superiores, apropiándose de ese beneficio.

Por otro lado, desde el punto de vista ambiental, el modelo industrial es extremadamente contaminante debido a que concentra una gran cantidad de animales en un espacio reducido de terreno. Esto tiene abundantes consecuencias negativas sobre el territorio, debido a que los deyecciones del ganado se concentran hasta tal punto que 
superan la capacidad de absorción del suelo. Además los animales criados en estas parcelas requieren una mayor cantidad de alimentos y otros recursos como agua (tanto para ingesta como para higiene), influyendo en la distribución y en la calidad de este bien natural. Otro aspecto muy importante que se debe tener en cuenta es la gran cantidad de desechos que genera la ganadería industrial. Según la FAO (2009), el sistema alimentario industrial descarta la mitad de la comida que produce en el transcurso de las distintas fases de la cadena de producción, es decir, desde el origen hasta los consumidores. Ya no es que esta cantidad sería suficiente como para alimentar a las personas hambrientas del mundo seis veces, sino que desde el plano ambiental, la pudrición de estos alimentos genera residuos además de emitir gases de efecto invernadero a la atmósfera.

Por último, es conveniente aclarar un aspecto. Se suele hablar de las elevadas eficiencia y productividad de la agricultura industrial en comparación con los sistemas de cultivo tradicionales que se encuentran, sobre todo, en el denominado Sur global (Grain, 2009). Sin embargo, esta expresión tan solo se refiere a términos económicos, ya que si se tienen en cuenta otros aspectos, como la eficiencia energética, la contribución en el sustento económico de las familias o la contribución al deterioro ambiental, las cuentas no saldrían. Por lo tanto, cabe plantearse una breve reflexión: la producción tiende cada vez más a cubrir las necesidades y los intereses del mercado en vez de buscar soluciones para diferentes problemas, como es el caso de la alimentación o la pobreza en el mundo, mediante un método sostenible. Esta tendencia mercantilista puede ser muy peligrosa para las producciones familiares, que no pueden hacer frente a las exigencias de este mercado elitista, y por ello, son rechazados por la gran distribución agroalimentaria. De no poner límite a esta tendencia, cientos de millones de personas que habitan en medios rurales perderán su modo de vida (y con ellos los agroecosistemas que formaban), generándose así más pobreza, más miseria y más migraciones a ciudades o lugares dónde los campesinos tampoco podrán encontrar una existencia digna.

\subsection{Influencia en los recursos naturales}

"El actual sistema alimentario mundial (donde se impone el modelo industrial), con todas sus semillas de alta tecnología y sus bonitos paquetes, no es capaz de cumplir con su función principal: alimentar a las personas" (Grain, 2009). Sin embargo, sí que ha contribuido a un deterioro del ambiente a gran escala y a que unas pocas corporaciones transnacionales se aseguren unos beneficios desorbitados.

Desde el punto de vista ambiental, el sector agroalimentario y todas las actividades que participan de él de manera indirecta influyen de manera decisiva en el deterioro del medio, tanto que se dice que este sector es uno de los principales motores del cambio climático (Grain, 2010). No obstante, el sector ganadero a nivel mundial es muy amplio. Representa el $40 \%$ de la producción agraria total y es la base de subsistencia de más de mil millones de personas. Además, no todas las actividades de este sector contribuyen de igual manera en el maltrato a la naturaleza. Con todo, debido a su estrecho vínculo con el territorio y con el ambiente, cabe destacar que bajo el punto de vista de los recursos naturales, según la FAO (2009), el sector pecuario es el mayor usuario y administrador de este tipo recursos en el mundo. Dicho sector ejerce una presión sobre el medio desproporcionada en relación con su importancia económica a escala mundial. Tanto es así que genera menos del $2 \%$ anual del PIB mundial, mientras que produce el 
$18 \%$ de los gases de efecto invernadero (FAO, 2009). Sin embargo, su importancia aumenta extraordinariamente si se tienen en cuenta otros criterios, como por ejemplo su papel capital en la seguridad alimentaria de todos los seres humanos del mundo. A continuación se expondrán de forma general algunas de las relaciones del sector ganadero con el medio, y más concretamente con los recursos naturales.

En primer lugar, respecto al uso del suelo cabe destacar que la ganadería utiliza actualmente el $30 \%$ de la superficie terrestre y el $80 \%$ de la superficie agrícola total (principalmente pastizales) (FAO, 2009). En este sentido, la actividad agropecuaria ocupa un tercio de toda la superficie cultivable solo para producir forraje. Un número importante de estas superficies han sido deforestadas de forma continua, particularmente en América del Sur. Un caso emblemático es el de la Amazonía, dónde el $70 \%$ de los bosques talados en ese gigantesco pulmón universal ha sido dedicado a pastizales (FAO, 2009). Dicho cambio en el uso del suelo supone una notable contribución a cuestiones ambientales tan trascendentales como el cambio climático. En la misma línea, es posible hallar otros problemas en los pastizales, como es el caso de su degradación debido a la sobreexplotación, además de favorecer su compactación y erosión, dificultando así su posterior aprovechamiento.

En segundo lugar, el sector pecuario también tiene un impacto notable en la calidad del agua. El agua es un recurso vital que además no es renovable. Sin embargo, la humanidad ha estado haciendo uso de él, en muchas ocasiones sin tener en cuenta las repercusiones negativas que podían tener esas actividades. El sector ganadero, como cualquier otra actividad humana, tiene su impacto en el ambiente y, por lo tanto, en el agua. El principal agente contaminante es el estiércol, que se vierte en los ríos por medio de los desagües, aunque también aparece por filtración en la tierra debido a la superación de la capacidad de carga del territorio. Estos procesos se dan sobre todo en las actividades pecuarias intensivas. La gestión del estiércol es un factor determinante a la hora de hablar de contaminación ambiental. Dicha gestión no solo afecta al agua, sino que también puede influir en otras cuestiones como las emisiones de GEI. Otro aspecto que se debe tener en cuenta en la relación producción agropecuaria-recursos hídricos es la fuerte competencia de uso que tiene este sector productivo con el uso humano. Esta competencia crecerá en los próximos años como consecuencia del crecimiento de la población mundial y al descenso de la cantidad de agua en condiciones óptimas para el consumo humano. En este sentido, ello supone un problema que hay que tener muy en cuenta. Por un lado, debido a la magnitud a escala planetaria del sector ganadero, y por otro, debido a que la escasez de agua, durante el transcurso de la historia, ha sido motivo de serios conflictos entre regiones y países.

Otro aspecto que es pertinente considerar, aparte de la producción, es la conversión de los alimentos en mercancías. Estos procesos dan como resultado un consumo elevado de energía fósil. Esta se emplea, entre otros usos, en el transporte de los alimentos alrededor del mundo, en su procesado, en su almacenaje, en los procesos de congelación $\mathrm{y}$ en la distribución hasta los hogares de quienes los consumen. Por supuesto, todos estos procesos contribuyen de manera determinante en la cuenta ambiental, además de que suponen un gasto desequilibrado de los recursos no renovables.

En este contexto, resulta necesario resaltar la influencia de la ganadería en la biodiversidad. El sector ganadero (sobre todo el modelo de producción industrial) busca la máxima rentabilidad por medio de la manipulación genética. Por ese motivo, en 
muchas ocasiones se favorece el cruce de razas y la creación de razas "cosmopolitas". Estas nuevas estirpes son creadas para su adaptación a la mayoría de los ámbitos en detrimento de las especies autóctonas, que son menos rentables en términos económicos. Por otro lado, la conversión de superficies forestales en pastizales o la monoespecialización en la creación de pastos y forrajes en detrimento de variedades de cultivo locales causa una pérdida de biodiversidad considerable.

\section{GANADERÍA Y CAMBIO CLIMÁTICO}

La producción agropecuaria se encuentra estrechamente interrelacionada con el medio y por lo tanto con el tan de moda cambio climático, en el que contribuye y del que la ganadería se ve perjudicada. A pesar del debate científico y político acerca de las causas antropogénicas o no del cambio climático, como ya se ha indicado, existe un consenso casi general en la teoría del calentamiento global como consecuencia de las actividades humanas. Estas actividades van ligadas al estilo de vida de los habitantes del planeta, que viene determinado por el sistema económico actual. En este sentido, es preciso destacar que la actividad ganadera es la actividad humana que más influye en la cuenta climática después del sector energético (Berra y Finster, 2002).

Las actividades humanas contribuyen a la crisis climática emitiendo gases de efecto invernadero (GEI) a la atmósfera. La ganadería, según la FAO (2009), aporta un 18 \% de estos gases lanzados a la atmósfera de forma general. Al analizar de manera más detallada la contribución total de la ganadería al cambio climático, se aprecia que sólo una pequeña sección de actividades pecuarias es responsable de casi todas las emisiones de GEI de todo el sector primario. La deforestación causada por el cambio de uso de la tierra es responsable de cerca de la mitad del total, aunque también es necesario tener en cuenta otras actividades imprescindibles para la producción o la distribución de los alimentos. En este sentido, según la FAO (2009), teniendo en consideración todo el conjunto de la cadena alimentaria, el ganado genera un $9 \%$ de las emisiones de dióxido de carbono antropogénicas, un $37 \%$ de las emisiones de metano y un $65 \%$ de las emisiones de óxido nitroso, que si se calculan en conjunto suponen el $18 \%$ de los GEI citados anteriormente. En relación con lo anterior, el modelo de producción que más contribuye en la cuenta climática es la ganadería intensiva, ya que su valor en producción y en uso de recursos naturales es superior.

Las principales fuentes emisores de GEI son tres: el uso de la tierra y los cambios en la utilización de la tierra (36\%), la gestión del estiércol (31\%) y la producción animal (25 $\%)$, mientras que la producción de piensos, la elaboración y el transporte no representan un porcentaje relevante ( $7 \%$ y $1 \%$, respectivamente). Sin embargo, a pesar de que el porcentaje sea menor, no se debe minimizar, pues sus cifras absolutas son muy altas. Como ejemplo ilustrativo, según la ONG Grain (2009), la Agencia de Protección Ambiental estadounidense informó que en 2005 la agricultura del país emitió tanto dióxido de carbono como 141 millones de automóviles juntos ese mismo año.

A partir de este desglose se observa en primer lugar la notable importancia que tiene el uso de la tierra. Desde el punto de vista del empleo de la tierra, cabe destacar que un mal empleo de esta superficie puede contribuir mucho a las emisiones totales de GEI. Por ejemplo, la práctica desmesurada de los desmontes para la creación de pastos y forrajes para el ganado elimina la capacidad de la tierra para actuar como sumidero de 
carbono que capture este gas de la atmósfera (FAO, 2009). Otro aspecto fundamental en la utilización del suelo es el pastoreo. Un pastoreo desmesurado puede causar una elevada nitrificación del suelo que propicie las emisiones de óxido nitroso.

En segundo lugar se encuentra la gestión de estiércol y la producción animal. Ambas pueden ser englobadas en un mismo grupo: el de las emisiones en las explotaciones ganaderas. Desde el punto de vista de la gestión del estiércol, estos desechos, por su constitución química, producen óxido nitroso $\left(\mathrm{N}^{2} \mathrm{O}\right)$, al igual que las plantas forrajeras como la alfalfa cuando se emplean en la nutrición del terreno. El óxido nitroso surge por la desnitrificación del nitrógeno contenido en el estiércol y en la orina del ganado. Por otro lado, como indican Berra G. y Finster L. (2002), a partir de la descomposición del estiércol también se producen emisiones de metano $\left(\mathrm{CH}^{4}\right)$ cuando éste se conserva en condiciones anaeróbicas, como por ejemplo en forma líquida en tanques o fosas. Sin embargo, las emisiones de este gas de efecto invernadero son escasas cuando el estiércol se descompone aeróbicamente, como sucede en la superficie de pastos y campos. En este aspecto también influye la temperatura a la que se produzca dicha descomposición, siendo las más propicias las temperaturas templadas. En este sentido, las emisiones de metano a partir del estiércol también dependen de la dieta del animal. Por supuesto, el manejo del estiércol constituye un asunto muy importante, ya que de su correcto uso se derivarán unas repercusiones positivas o negativas para el terreno. La emisión de GEI es tan solo una consecuencia ambiental más de las que puede tener la gestión de este subproducto.

El otro pilar de las emisiones en las explotaciones ganaderas proviene de la producción animal. Se trata de la denominada fermentación entérica, que es un proceso más de la digestión animal. La emisión de metano se produce a través del exhalado o el eructado del animal. Dicho proceso tan solo se da en los rumiantes debido a que son poligástricos. En el caso de los animales no rumiantes la fermentación microbiana se da en el intestino grueso y no en el rumen, por lo que la producción de metano es mucho menor (Berra y Finster, 2002). Este dato hay que tenerlo muy en cuenta, ya que indica que las emisiones de vacas y búfalos son mayores que las de otros animales como los cerdos y las aves de corral. En este sentido es preciso mencionar que el metano, el principal GEI emitido por la ganadería, tiene un potencial de calentamiento veintitrés veces superior al $\mathrm{CO}^{2}$. Una vaca lechera produce, de forma natural, aproximadamente $75 \mathrm{~kg}$ de $\mathrm{CH}^{4}$ al año, que equivale a más 1,5 toneladas de $\mathrm{CO}^{2}$. Asimismo, baste mencionar como ejemplo que en América Latina y Asia las emisiones producidas por los rumiantes suponen el $85 \%$ de las emisiones del sector, sobre todo en forma de metano (FAO, 2009).

La producción de piensos también causa un porcentaje de emisiones relevante, sobre todo la utilización de combustibles fósiles. Una vez más habría que plantearse algunas cuestiones acerca de la rentabilidad energética de la producción de pastos, forrajes y cereales para la alimentación de los animales. Otra cuestión sería plantearse si es "moralmente" correcto que existan millones de hectáreas de cereal dedicadas al alimento del ganado (cuya carne es consumida tan solo por sectores pudientes, y en ocasiones se desecha), mientras millones de personas se mueren, literalmente, de hambre. Este aspecto se abordará con mayor profundidad más abajo.

Por último, el sacrificio de los animales y la elaboración y transporte de los productos resultantes también producen emisiones. Éstas están relacionadas con el empleo de 
combustibles fósiles y el desarrollo de las infraestructuras. En este sentido, se debe tener en cuenta la totalidad del entramado agroindustrial que procesa el alimento, lo refrigera y lo empaca. Del mismo modo se ha de considerar el tipo de materiales que se utilizan para realizar estos procesos, así como los combustibles y los desechos generados.

\subsection{Cómo influye el cambio climático en el sector agropecuario}

El vínculo que se establece entre la producción agropecuaria y el ambiente depende de factores locales, como el clima, la configuración del terreno, las características agrológicas, las condiciones económicas, las técnicas que predominan en la producción o el tipo de explotación (Segrelles, 2010). Por lo tanto, todas las modificaciones que tengan lugar en el sistema climático afectarán seriamente a los agroecosistemas actuales y por consiguiente a la seguridad alimentaria. Como era de esperar, los efectos del cambio climático no son iguales en todos los lugares del globo, sino que vienen dados por la latitud del lugar en el que se desarrollen las explotaciones. Estos efectos producen cambios en factores como la temperatura y la humedad, que condicionan el alojamiento, los pastos y cosechas para la alimentación del ganado, entre otros.

A la hora de abordar esta problema, una vez más, se debe distinguir entre el mundo desarrollado y el subdesarrollado ya que las diferencias en los estilos de vida y consumo conllevan unas consecuencias ambientales diferentes. Por ejemplo, como ya se ha hecho mención, para la simple puesta en circulación de los alimentos son necesarios unos procesos industriales que causan determinados efectos en el medio (De Cuenca, 2008). Las grandes ciudades necesitan de un abastecimiento continuo concentrado en un periodo o días muy concretos de la semana, lo que conlleva todo un proceso de fabricación e introducción de los alimentos en los canales comerciales. Por otro lado, el grado de desarrollo de las infraestructuras y la capacidad de inversión facilitarían la adaptación frente a las posibles adversidades.

En el lado opuesto se halla el llamado Tercer Mundo. Resulta muy difícil hablar del asunto que aquí se estudia en este ámbito planetario. En estas áreas y según los diferentes lugares, el agua y los pastos escasean. Además se reciclan los residuos animales a través de las explotaciones tradicionales que aún respetan la relación tierraexplotación. No obstante, es cierto que existen zonas donde sí se podría hablar de sobreexplotación y contaminación, aunque éstas vienen definidas, en la mayoría de los casos, por la invasión de las estrategias neoliberales. A pesar de todo resulta peliagudo querer hablar de desarrollo sostenible y limitación de las emisiones en lugares donde todavía no se ha iniciado el citado desarrollo. Sin embargo, y pese a la escasa contribución en la debacle mundial, las áreas rurales de los países menos desarrollados serán las más afectadas debido a su escasa capacidad de adaptación por falta de capital.

Lo anteriormente expresado halla su explicación en que los efectos del cambio climático en el sector agropecuario serán diferentes según se trate de ganadería extensiva o intensiva. La ganadería extensiva, como se ha podido observar, es la que se encuentra más ligada a las condiciones naturales del medio, por lo que se verá más afectada directamente por las modificaciones ambientales que se produzcan. El modelo tradicional extensivo tiene que sobrevivir en la actualidad luchando contra unas condiciones poco propicias. Lo peor es que las oportunidades que les depara el futuro, según los expertos, son escasas o nulas. Por ejemplo, según la FAO (2009), desde el 
punto de vista de la alimentación del ganado, el aumento de las temperaturas influirá en que en las zonas áridas o semiáridas el ganado ingiera menos alimento. Su producción, por lo tanto, será menor, dando lugar a una situación de estrés fisiológico. En cuanto a los pastos y los forrajes, la ausencia de precipitaciones hará que los pastos se desarrollen más lentamente. De este modo, la sobreexplotación y la degradación serán mayores. En estrecha relación con esto aparecerán problemas de acceso y necesidad de agua, problema que será compartido con los seres humanos. La ausencia de alimento y agua pueden desencadenar enfermedades en los animales que afecten a su productividad cárnica y a su fertilidad.

Las condiciones directas del clima apenas se dejarán sentir en los sistemas productivos intensivos o industriales, aunque sí que padecerán sus consecuencias de manera indirecta (FAO, 2009). Un ejemplo de estas consecuencias indirectas es el aumento del precio del cereal por la competencia con su uso para la elaboración de biocombustibles ${ }^{8}$, un nuevo uso que esta siendo muy potenciado a raíz del problema climático. Asimismo, el aumento del precio de la estabulación debido al consumo energético para refrigeración también es otra consecuencia indirecta que puede repercutir en el modelo intensivo. En otro sentido, para hacer frente a las consecuencias que el clima pueda tener sobre las especies animales, el modelo intensivo es capaz de escoger las razas que genéticamente les sea más favorable según criterios productivistas y de adaptación al clima. Esto supondrá una pérdida de biodiversidad a cambio de productividad y rentabilidad económica. Ello no representará ninguna novedad, ya que en la actualidad el modelo de explotación intensivo emplea, en su mayoría, razas cruzadas. Es por ello que un cambio en los factores climáticos contribuirá a la búsqueda de nuevas estirpes que se adapten a las nuevas condiciones en detrimento de las razas autóctonas. Son lo que en etnología se conocen como "razas cosmopolitas",.

Estas son algunas de las dificultades a las que tendrá que hacer frente la ganadería. Por un lado se aprecia cómo el modelo extensivo tendrá que malvivir y aguantar hasta que pueda, mientras que, por otro lado, el modelo intensivo se adaptará mediante una gran inversión y un gran consumo de energía para de este modo crear microclimas en sus explotaciones, además de manipular genéticamente los animales. En conclusión, el que sea capaz económicamente de adaptarse a las adversidades climáticas saldrá claramente beneficiado, pues los productores que no puedan invertir en ello, no sobrevivirán y cederán su cuota de mercado. En relación con estas cuestiones se puede afirmar que el cambio climático puede mermar la producción de alimentos en el mundo en desarrollo, mientras que los países industrializados (o el mundo desarrollado, siendo indiferente el lugar donde se produzca) pueden ganar en potencial de producción, según afirmó el Director General de la FAO, Jacques Diouf, en una intervención en la Fundación M. S.

\footnotetext{
${ }^{8}$ Alternativa que surge en contraposición a la subida del precio del petróleo. En muchos casos también se relaciona con una búsqueda de combustibles menos contaminantes. Algunos países, como los miembros de la UE, pretenden alcanzar una cuota de entre 10-20\% en el uso de biocombustibles. Al no contar con superficie suficiente para el cultivo del cereal necesario para estos porcentajes, las empresas bioenergéticas se deslocalizan a otras regiones, sobre todo tropicales, para desarrollar cultivos como el aceite de palma. Todos estos procesos tienen consecuencias ambientales (por ejemplo, la deforestación) y socioeconómicas (por ejemplo, habitantes del medio rural forzados al desplazamiento).

9 En este caso se aplica a las razas animales que se han generado como consecuencia del cruce de diferentes razas autóctonas de diversos lugares. El fin de estos cruces es conseguir una mayor adaptación a las condiciones climáticas del lugar donde se vaya a dar la explotación, y con esto una mayor y más rentable productividad.
} 
Swaminathan en Chennai, India." (FAO, 2007).

En último lugar es necesario hacer una especial mención a las variaciones que se producirán en el mapa de las enfermedades como consecuencia del cambio climático. Éste es un aspecto que afectará tanto a los seres humanos como a los animales, pertenezcan o no al mundo ganadero. Por un lado, como era de esperar, el cambio en las pautas climáticas provocará un desplazamiento de los ámbitos climáticos de diferentes enfermedades en relación con la modificación del hábitat de diferentes insectos portadores de enfermedades. Varias de estas enfermedades afectarán al ganado, y luego llegarán a transmitirse muchas veces a los seres humanos. Por supuesto, las regiones más pobres serán las que más lo padezcan, ya sea a través del contagio de las personas o a través del contagio de su medio de supervivencia (ganado). Esto es así debido a que la capacidad adquisitiva de las personas será la que determine el acceso o no a los medicamentos, tanto para los seres humanos como para los animales. Del mismo modo, la imposibilidad de combatir estas enfermedades creará dificultades para poder salir de la pobreza.

En este sentido también es necesario advertir que a pesar de que en la actualidad las grandes empresas farmacéuticas disponen de un arsenal medicinal suficiente para paliar muchas enfermedades, el cambio del ámbito territorial puede desembocar en modificaciones concretas de determinados virus (FAO, 2009). Esta cuestión debe ser tenida en cuenta ya que en muchas ocasiones los grandes centros de producción agropecuaria se concentran en las proximidades de los grandes asentamientos urbanos.

Por último, si se contempla el problema bajo otro punto de vista, el ganado también ofrece la posibilidad de ejercer en algunas zonas, una función de saneamiento público porque consume productos de desecho que, de otro modo, supondrían un grave problema de contaminación y salud pública.

\section{EL APROVECHAMIENTO GANADERO: UN SECTOR INJUSTO Y CONTRADICTORIO}

Las ideas neoliberales han alcanzado en la últimas décadas al sector agropecuario, transformándolo de forma radical. El modelo industrial trajo consigo un aumento extraordinario de la producción por medio de la introducción de mejoras técnicas y de manejo. Sin embargo, como ya se ha destacado, no ha conseguido su principal cometido: alimentar a la población, mientras que sí ha generado graves consecuencias de tipo ambiental. Por eso es preciso destacar que en los últimos años el crecimiento de la producción animal ha sido tres veces mayor que el crecimiento demográfico, y que en la actualidad se ha llegado a conseguir un excedente de más de tres veces y media la población mundial. A pesar de esto, a día de hoy casi mil millones de personas en el mundo (un $16 \%$ aproximadamente de la población mundial) padecen problemas de desnutrición (FAO, 2009).

En una economía capitalista el acceso a los diferentes bienes y servicios viene determinado por la capacidad adquisitiva del demandante de éstos. Asimismo, la FAO (2009), indica que "la seguridad alimentaria no depende del suministro, sino de la capacidad adquisitiva de la demanda". Dicha idea se puede percibir si, por ejemplo se observa que de los mil millones de seres humanos que padecen desnutrición, el $95 \%$ se 
encuentra en países en vías de desarrollo y dentro de éstos sobre todo en las zonas rurales (FAO, 2005). Del mismo modo, existen diferencias notables en el consumo per cápita de carne. Según los datos de la FAO (2005), se observa un desequilibrio entre los $82 \mathrm{~kg}$./hab./año que se consumen en los países desarrollados y los 30,9 kg./hab./año del mundo empobrecido. Estas singulares diferencias son las que explicarían que mientras en el mundo empobrecido las enfermedades más comunes son las relacionadas con la falta de alimentos, en el denominado primer mundo las enfermedades más comunes son las de tipo cardiovascular y la obesidad, relacionadas ambas con el exceso en el consumo de carne (FAO, 2009). Por consiguiente aparece en el mundo desarrollado un sector de la población, cifrado en 1.600 millones de personas, con problemas de sobrepeso, frente a los mil millones de habitantes que carecen de alimentos.

Esta situación afianza aún más la teoría de que el sector agroindustrial busca alcanzar otro tipo de objetivos lejanos al de abastecer a la población, es decir, sí que busca abastecer a población, pero a una población que pueda pagar y reportarle beneficios económicos. En este caso se puede tomar en consideración el caso de Argentina. El 57 $\%$ de los productos que este país vende al exterior son productos alimentarios. Estos productos reportan al país aproximadamente 30.700 millones de dólares. Mientras tanto, según el Ministerio de Salud (1996), un 13\% de los niños padece desnutrición. Algunos expertos, como Alejandro O'Donnell (director del $\mathrm{CESNI}^{10}$ ), sitúan la sitúan este porcentaje en el $20 \%$. Sin embargo, esta situación parece no preocupar a los expertos en agro-negocios que prefieren por apostar por un crecimiento del país como proveedor de alimentos y materias primas agroalimentarias (Pérez Satisteban, 2007). Es más, también es preciso resaltar otras afirmaciones como por ejemplo la voluntad de instalar la exportación argentina en los segmentos globales de mayor poder adquisitivo, "donde la demanda es mucho menos sensible al precio, el consumo es estable, la rentabilidad es más alta y las cantidades demandadas se enmarcan en volúmenes muy adecuados a la capacidad argentina" (Vanella, 2007). En relación con lo anterior cabe destacar que la población más pobre de los países exportadores de piensos y carne, localizadas sobre todo en las zonas rurales, no solo no tienen capacidad para adquirir estos alimentos cárnicos, si no que ven cómo los usos tradicionales que les proporcionaban su sustento se ven forzados al abandono.

Por lo tanto, debido a la pretensión de alcanzar objetivos puramente económicos, el sector ganadero ha dejado de lado su función como proveedor de alimentos y su responsabilidad como actividad que influye y condiciona el medio, rompiendo así el equilibrio natural. Desde el punto de vista ambiental, la actividad agropecuaria industrial es un ejemplo de producción completamente desequilibrada. Como ya se ha indicado, el modelo ganadero industrial busca una producción con costes económicos muy bajos y en un periodo de tiempo muy corto. Por el contrario no se plantea otro tipo de desajustes, como sucede por ejemplo con el derroche de los recursos naturales.

Ya se ha destacado la extensa superficie que ocupa el cultivo de cereales, tanto para el consumo animal como para la creación de biocombustibles. En relación con esto, también se ha hecho mención de que algunos sectores, como la selva ecuatorial, se ven deforestados para cambiar su uso y cultivar estos cereales, con unas consecuencias

\footnotetext{
${ }^{10}$ Centro de Estudios sobre Nutrición Infantil “Dr. Ajejandro O’Donnell”. Es una entidad de Bien Público fundada en 1979 que tiene como propósito hacer operativo el conocimiento para promover una mejor nutrición infantil.
} 
catastróficas sobre el ambiente. Dichos cereales son, en gran medida, vendidos como materias primas a empresas de otros países que las emplearán como alimento para el ganado, que aporta mayores ingresos. En este sentido, según los datos que aparecen en el documental Home (2010), el $50 \%$ de la producción de cereal se utiliza para la producción animal y de biocombustibles. Una relación que se vuelve más impactante si se considera que Estados Unidos cosecha cantidades suficientes de cereales para alimentar a 2.000 millones de seres humanos, solo que estos en su mayoría van a parar a la industria ganadera y bioenergética (HOME, 2010).

La gran distribución agroalimentaria necesita de un excedente animal a partir del cual seleccionar productos destinados a la venta y otros a la pudrición, llegando a perderse casi la mitad de la producción animal. Dicha producción podría alimentar a toda la población necesitada de sustento. Un aspecto relacionado con este asunto y que debe llamar la atención es la cantidad de pienso que se necesita para conseguir un kilogramo de carne. Por ejemplo, para la producción de un kilogramo de carne de res se necesitan 10 kilogramos de cereal, mientras que se necesitan dos para uno de pollo y 4 kilogramos para conseguir uno de queso o un litro de leche (Santamarta, 2007). En este sentido, gracias a estos datos se puede reflexionar sobre la necesidad de un cambio en las pautas de consumo mundial, reduciendo el consumo de carne en general, sustituyendo la carne de vacuno (que por otro lado también contribuye más a la emisión de gases de efecto invernadero) por carne de pollo, además de emplear ese cereal en el consumo humano ${ }^{11}$.

Otro aspecto destacable de este modelo ganadero desequilibrado (modelo industrial) que se intenta imponer a nivel global es su relación con el agua. Ya se ha planteado esta relación en el epígrafe "Ganadería y ambiente», aunque con el fin de resaltar más las incongruencias de este sector, es preciso exponer algunas relaciones dialécticas del sector ganadero con los diferentes recursos naturales. Por ejemplo, desde el punto de vista del uso de agua, ya se ha comentado su situación como recurso vital, no renovable, y su situación de escasez y deterioro que podría desembocar en futuros conflictos relacionados con las luchas por su aprovechamiento. La ganadería consume una proporción hídrica muy importante dentro del sector primario (el sector primario en general consume un $70 \%$ del recurso agua). Según la Comisión de la ONU sobre Desarrollo Sostenible,son necesarios hasta 7.000 litros de agua para producir 100 gramos de carne de res (Santamarta, 2007). A modo de ejemplo también se podría destacar que la creación de una ración de pan hecha con harina solo necesita 550 litros de agua. Siguiendo esta relación, para producir un kilogramo de carne de res se necesitaría más cantidad de agua que la que emplea una persona de media en su aseo personal diario durante un año (Santamarta, 2007). Así, se ahorraría mucha más agua reduciendo la proporción de carne en la dieta que consumiendo menos agua en el aseo personal.

Otro aspecto muy relevante es el consumo de energías fósiles, principalmente petróleo. El sector agropecuario consume este recurso sobre todo a partir de actividades no relacionadas con su producción, sino con su distribución y conservación. De este modo, en el proceso que se desarrolla desde la fabricación del producto cárnico hasta la llegada del mismo a los frigoríficos de los grandes supermercados se consumen grandes cantidades de energía. Este ciclo comenzaría con el cultivo de los cereales para

${ }^{11}$ En 1990, el programa de hambre en el mundo de la Brown University estimó que los cultivos mundiales podrían suministrar una dieta vegetariana a 6.000 millones de personas si fueran distribuidos equitativamente y sin aportar esa importante proporción a la alimentación del ganado. 
alimentar al ganado vacuno. En este caso, el cultivo requiere del uso de productos químicos agrícolas derivados del petróleo. Posteriormente hay que añadir el combustible requerido para el transporte continuo de este pienso a las instalaciones ganaderas, así como el traslado del ganado vacuno a los mataderos, y desde allí a los mercados, recorriendo miles de kilómetros. En relación con esto, los procesos de conservación o de empacado (mediante productos que muchas veces son derivados del petróleo) también suponen un gasto importante de energía (Santamarta, 2007). Como ejemplo se puede destacar que, según el director de World Watch, J. Santamarta Flórez, se requieren 8,3 litros de gasolina para producir un kilogramo de carne de res alimentada con pienso en Estados Unidos; del mismo modo que se requieren 28 calorías de energía de combustibles fósiles para producir una caloría de proteína de carne para el consumo humano, frente a las 3,3 calorías de combustible fósil para producir una caloría de proteína de cereal (Santamarta, 2007). Otro dato significativo relacionado con esta cuestión es que, por término medio, la agricultura de los países industrializados gasta cinco veces más energía comercial (gas y combustible fósil) para producir un kilogramo de cereal que la africana (Grain, 2009).

Desde el punto de vista de la generación de residuos, un uso ganadero industrial que no se hace responsable de su papel en el medio genera unas cantidades enormes de residuos. Según J. Santamarta (2007), esta generación de residuos puede superar en 130 veces los generados por la población. Estos residuos de origen ganadero, como ya se ha comentado, pueden alcanzar los acuíferos y los ríos causando la contaminación de sus aguas, contaminación que puede llegar a afectar a las superficies marinas ${ }^{12}$.

Al hilo de lo comentado, cabe plantearse una reflexión. La población mundial alcanzará 7.000 millones de individuos en un periodo breve de tiempo y crecerá aún más en las próximas décadas. Esto supone un reto para el equilibrio global. Por otro lado, si continúan las tendencia actuales, el planeta, tanto en su vertiente física como en su vertiente humana, sufrirá unas terribles consecuencias. Todo indica que pese a la existencia de contados intentos, las pautas que se van a seguir serán las mismas. Los patrones de desarrollo establecidos por el capitalismo ahora son empleados tanto por las firmas transnacionales como por las empresas nacionales en los nuevos países desarrollados. Estos países acogen, con buenas esperanzas para su futuro económico, modelos productivos basados en una nula conciencia ambiental. En este sentido, estas nuevas economías desarrolladas (los casos más significativos serían China e India) han sido las que han impulsado la mayor parte del crecimiento mundial en los últimos años. Este crecimiento va unido a un aumento de la proporción de la clase media, y por consiguiente una búsqueda de unas mejores condiciones de vida que depararán un consumo de recursos y un consecuente deterioro ambiental sin precedentes. Por su parte, la copia de los modelos culturales y de comportamiento exportados desde regiones desarrolladas, como Estados Unidos, generarán (siguiendo el binomio producción-consumo) unos patrones de consumo y de producción de carne claramente insostenibles que superarán los límites naturales establecidos. Desde el punto de vista del mundo subdesarrollado, la competencia por la explotación de los recursos escasos, unida al aumento de la población y a la definitiva consolidación del modelo industrial

\footnotetext{
${ }^{12}$ Los nutrientes que aparecen en la composición de los residuos ganaderos motivan el desarrollo de las algas, que consumen el oxígeno del agua, creando una "zona muerta" en el Golfo de México. En esta zona muerta no hay suficiente oxígeno para permitir la vida acuática (Santamarta, 2007).
} 
frente a las explotaciones tradicionales, traerá consigo un aumento de las condiciones de vida negativas que existen en la actualidad (especialmente la pobreza, el hambre y la miseria), es decir, una acentuación del clásico desequilibrio mundo desarrollado-mundo en desarrollo. Sin embargo, este problema humano, que en principio puede afectar a unos y beneficiar a otros, se convertirá en un fenómeno negativo a escala global, que afectará tanto a los ricos como a los pobres (cambio climático).

\section{CONCLUSIONES}

Pero las estadísticas confiesan. Los datos ocultos bajo el palabrerio revelan que el veinte por ciento de la humanidad comete el ochenta por ciento de las agresiones contra la naturaleza, crimen que los asesinos llaman suicidio, y es la humanidad entera quien paga las consecuencias de la degradación de la tierra, la intoxicación del aire, el envenenamiento del agua, el enloquecimiento del clima y la dilapidación de los recursos naturales no renovables.

Eduardo Galeano

El sistema agroalimentario actual resulta ineficaz, pero no solo porque no ha conseguido alimentar a toda la población del planeta, sino por el gravísimo coste ambiental que ha tenido. Se ha pasado (o se pretende pasar) de un modelo tradicional equilibrado con el ambiente a un modelo industrial muchísimo más productivo pero igual de eficaz, y que además es muy perjudicial para el medio. La causa principal de esta ineficacia, no reside tanto en el mal empleo de los recursos o en la baja producción, sino más bien en que éstos son solo una consecuencia más de su razón de ser, es decir, de sus objetivos capitalistas. El objetivo principal del sector agropecuario, controlado por la gran distribución, no es alimentar a toda la población, pues lo que más le preocupa es conseguir el máximo beneficio en el periodo más corto de tiempo posible. Si su objetivo real fuera suministrar alimento a la población, éste se podría llevar a cabo sin grandes problemas.

Todo ello va ligado al poder de las grandes distribuidoras que controlan la totalidad de la cadena alimentaria. Ellas, al controlar la distribución, influyen de manera determinante en los procesos de producción y consumo, llevándose los beneficios reales. Ante esta situación, el reducido tamaño productivo de algunos ganaderos motiva que éstos se vean sometidos a las condiciones asfixiantes que impone la gran distribución. En definitiva, la gran distribución ha alcanzado un poder que supera en muchas ocasiones al de los estados. Este poder lo usa en su propio beneficio, al mismo tiempo que genera graves problemas humanos y ambientales. En este sentido, por qué no se podría relacionar la actuación de estas grandes empresas transnacionales con los actos inhumanos de los dictadores europeos del siglo XX (Santamarta, 2007). Esta comparación no sería descabellada. Las grandes transnacionales de la distribución alimentaria niegan un alimento existente a la mayor parte de la población porque no consiguen ningún beneficio con ello. De esta manera se le niega el alimento (necesario para vivir) a más de 1.000 millones de personas. Cabe recordar que en el año 1948, en la 
Declaración de los Derechos Humanos, se estipuló como derecho humano fundamental el derecho a la alimentación. Ésta sería una de las consecuencias directas que se están dando a corto plazo. Asimismo, habría que considerar las consecuencias humanas del futuro cambio ambiental.

Los diferentes problemas planteados en esta reflexión no afectan de la misma manera a todos los habitantes del planeta. Una vez más, los sectores más pobres serán los que más se vean afectados por los futuros problemas ambientales. Éstos se añadirán a sus actuales problemas de hambre y miseria. Asimismo, el medio rural tradicional (al contrario del industrial) será el espacio humano más afectado debido a su estrecho vínculo con el medio natural. Dichas consecuencias le vendrán dadas por usos productivos y de consumo a los que el medio rural tradicional permanece ajeno. La explicación se encuentra en el carácter global del medio y en las interrelaciones que se establecen entre sus diferentes elementos. Las consecuencias naturales vendrán establecidas como consecuencia de los factores humanos, mientras que serán repartidas por todo el territorio como consecuencia de procesos naturales, como por ejemplo la dinámica atmosférica.

Todavía existen controversias acerca de las causas del actual cambio climático. Sin embargo, en este artículo se apoya la teoría del calentamiento global por «efecto invernadero». Dicha teoría expone que el ser humano, mediante sus actividades, esta provocando el mencionado cambio. Asimismo, si se continúa con este deterioro, las previsiones no deparan un futuro muy alentador para la vida humana. En esta línea, por ejemplo, según se extrae del documental Home (2010), el aumento de las temperaturas del planeta puede provocar un deshielo del permafrost de Siberia, produciendo una liberación de cantidades elevadas de metano. Una situación que conduciría a un aumento en la proporción de metano $\mathrm{CH}^{4}$ en la atmósfera y a la creación de una situación física en la tierra donde el desarrollo de la vida humana sería imposible. Con todo, y aunque estas previsiones futuras no sean exactas, es evidente que el ser humano, mediante sus actividades productivas, está degradando extraordinariamente el medio, por lo que, de seguir así, no nos cabe esperar un futuro muy alentador.

El papel representado por los gobiernos también ha sido fundamental en este problema. La asimilación de la teoría del calentamiento global por efecto invernadero llevó a diferentes naciones a acordar una serie de medidas para hacer frente a este fenómeno. Estas medidas propuestas fueron recogidas en el Protocolo de Kyoto del Convenio Marco sobre Cambio Climático de la ONU (UNFCCC) ${ }^{13}$. Dicho acuerdo supuso un primer paso para hacer frente a la cuestión climática a escala transnacional. Sin embargo "Kyoto" no está libre de polémicas y controversias, tanto a nivel científico como a nivel político $^{14}$. Según opina P. Bidwai (2007), se trata de un acuerdo demasiado modesto, pues solo se propuso reducir las emisiones un 5,2 \% para el año 2012, en lugar de los 25-30\% necesarios para prevenir la catástrofe. Lo peor de todo es que después de una década de mensajes políticos bienintencionados no se ha conseguido alcanzar siquiera la

\footnotetext{
${ }^{13}$ El objetivo del protocolo de Kyoto es conseguir reducir, para el periodo 2008-2012, un $5 \%$ las emisiones de gases de efecto invernadero globales sobre los niveles de 1990.

14 Según diferentes estudios, para influir realmente en el cambio climático se deberían reducir las emisiones en unos niveles muy superiores a los propuestos por el Protocolo de Kyoto. Por otro lado, existe países que apuestan por reducir aún más sus emisiones (Ej. países de la Unión Europea) y países muy influyentes en la cuestión y que hasta hace poco no estaban de acuerdo con el Protocolo de Kyoto (Ej. Estados Unidos).
} 
cifra de 5,2 \%. Los motivos se pueden resumir en dos cuestiones. Por un lado, en muchas ocasiones, el poder de la gran distribución es tan grande que lo estados a penas pueden intervenir dentro del sistema actual, y por otro lado, resulta evidente que los diferentes países también están en competencia constante, por lo que sus objetivos se basan también en un crecimiento económico continuo.

Tras esta recapitulación es preciso exponer una serie de propuestas a pequeña escala que contribuyan a paliar la crisis climática $\mathrm{y}$, en definitiva, los problemas derivados del sistema productivo actual.

En primer lugar, como indica la ONG Grain (2009), el alimento se encuentra en el centro de la actual crisis climática. Lo que también es cierto es que la producción de alimentos deberá estar al día con las variaciones demográficas que se produzcan en los próximos años. Por eso, los modelos actuales no se pueden repetir, pues son claramente injustos e irresponsables. Para ello, el primer y más importante paso es la necesidad de cambiar la mentalidad, tanto la de la gran distribución que controla la cadena alimentaria como la de los productores y consumidores que participan en ella. Debemos eliminar el pensamiento competitivo y crear una "economía solidaria de toda la casa" (Fernández Steinko, 2010).

Es necesario un cambio radical que sustituya el actual modelo insolidario de acumulación por otro modelo basado en la búsqueda de la dignidad de la vida de todos los seres humanos y en el respeto en su sentido más amplio, es decir, que a partir de él se produzca la solidaridad con un medio natural limitado y con las personas menos favorecidas. Para conseguirlo se debe dejar de valorar todo desde criterios únicamente económicos, de explotación y competitivos, y empezar a considerar otros criterios, como el ecológico o el humano, así como la cooperación y la ayuda mutua. En definitiva, resulta de vital importancia establecer unas pautas de comportamiento viables que apunten hacia una sociedad más justa y sostenible y que no se centren en generar una mayor calidad de vida para unos pocos, sino que ante todo se dediquen a buscar la dignidad de todos.

Para ello, desde el punto de vista del consumo, es necesario que el consumidor sea consciente de sus actos, sabiendo que, debido al carácter global del medio, lo que se hace en un lugar de la tierra puede repercutir en la vida de otras personas y otros territorios. El papel de la sociedad también es fundamental. No todos tienen la misma capacidad de actuar. En consecuencia, desde el denominado primer mundo se debe tomar la iniciativa y ser los primeros en no tolerar esta situación, al mismo tiempo que se renuncia a un estilo de vida innecesario e insostenible basado en la degradación ambiental y la miseria de millones de personas. Resulta obligatorio abandonar la pasividad mental impuesta por un estilo de vida consumista acorde con el sistema. Hoy en día el acceso a la información sobre este y otros temas es gratuito y libre, a través de medios como Internet. Por este motivo ya no existe ninguna excusa para no actuar en consecuencia. Así, merece la pena destacar una cita del famoso científico alemán, A. Einstein: "El mundo es un lugar peligroso. No por causa de los que hacen el mal, sino por aquellos que no hacen nada por evitarlo".

Desde el punto de vista de la producción y la distribución la situación planteada tiene una solución sencilla. La sociedad actual cuenta con suficientes avances técnicos y científicos para cambiar el rumbo de las cosas. Sin embargo, los sectores que controlan 
la cadena alimentaria se niegan a ceder su cuota de poder aun conociendo los problemas comentados. Ese es el principal freno, siempre y cuando estas grandes firmas transnacionales no descubran la forma de sacar beneficio del calentamiento global. Por lo tanto, si se quiere paliar la crisis climática, también llamada "crisis de civilización", se debe descentralizar ese poder.

Otro aspecto que se debe tener en cuenta sería la retirada de la producción alimentaria del ámbito de la OMC. Este tema ya fue reivindicado por la organización internacional La Vía Campesina en 2009. La producción de alimentos basada en criterios mercantilistas ha traído consigo unas consecuencias ambientales y humanas negativas. Abandonando los criterios de "bajo coste-máximo beneficio" se podría alcanzar una ganadería sostenible, que elaborara productos de calidad y que sirviera como medio de vida digno para los ganaderos.

En relación con esto, se debe resaltar la función trascendental que tiene el ganadero. El ganadero como productor individual, hoy por hoy resulta más vulnerable para verse sometido a las condiciones de las grandes distribuidoras y el mercado. Por lo tanto, una salida a este problema sería la agrupación de pequeños productores mediante cooperativas con el fin de tener una mayor capacidad de decisión y de gestión frente a organismos u entidades de mayor tamaño. Es importante el significativo papel que desempeñan las cooperativas agrarias en la producción ecológica (pues está conformada por pequeños productores que emplean métodos poco industrializados) y en el desarrollo sostenible del medio rural.

En este artículo se han expuesto las principales contribuciones de la industria ganadera, como actividad humana, al deterioro ambiental. Del mismo modo, se ha expuesto lo necesaria que es esta actividad, tanto para el alimento de los seres humanos como para el sustento de cientos de millones de personas. Con todo, no se debe considerar la ganadería como un sector que solo tiene aspectos negativos, ya que, como se ha visto, puede tener consecuencias positivas y negativas, e incluso ambas al mismo tiempo (Segrelles, 2010). Por ello resulta conveniente reflexionar sobre la insostenibilidad de nuestros estilos de vida y pautas de consumo y acerca de si son apropiados los métodos empleados en la producción agropecuaria.

\section{BIBLIOGRAFÍA}

AFP, DPA y REUTERS. FAO: 6 millones de niños mueren por hambre $y$ $\begin{array}{llll}\text { desnutrición al año. } & \text { [en } & \text { línea]. }\end{array}$ http://www.jornada.unam.mx/2005/11/23/046n1soc.php, [26 de noviembre de 2010].

BARROS, V. Cambio climático global. Buenos aires. Libros de la Zorzal. 2006. 174 p.

BERRA, G. y FINSTER, L. Emisión de gases de efecto invernadero; la influencia de la ganadería argentina. Cadena de la Carne Vacuna, Tecnologías para nuevos escenarios, IDIA 21 (2). 2002. p. 212-215. 
CASAL, M. Consecuencias de la dependencia del petróleo en la agricultura. Soberanía Alimentaria. Biodiversidad y Culturas. nº 3. Barcelona. 2010.

CRETTAZ, J. Será récord este año la exportación de alimentos. Diario La Nación. [en línea]. Buenos 2007. $<$ http://www.lanacion.com.ar/nota.asp?nota_id=973754>, [27 de diciembre de 2010].

FAO. El estado mundial de la agricultura y la alimentación. La ganadería, a examen. Organización de las Naciones Unidas para la Agricultura y la Alimentación. Roma. 2009.

FERNANDEZ STEINKO, A. Cambio del modelo productivo. Hacia una economía solidaria de toda la casa. El Viejo Topo. I. 2010. p. 44-51.

GAUDÍN G. Agricultura a gran escala afectan equilibrio ecológico. Noticias Aliadas. Cambio climático: Seguridad Alimentaria, agua \& protección de bosques. Buenos Aires. 2010.

GRAIN. Campo y crisis climática. Soberanía Alimentaria. Biodiversidad y Culturas. ${ }^{\mathrm{o}}$ 1. Barcelona. 2010.

Informe Cambio Climático 2001. Grupo de trabajo II. Grupo Intergubernamental de Expertos sobre el Cambio Climático. Accra. 2001.

Informe Cambio Climático 2007. Grupo de trabajo II. Grupo Intergubernamental de Expertos sobre el Cambio Climático. $<$ http://www.mma.es/secciones/biblioteca publicacion/publicaciones/revista ambie nta/n66/pdf/06informe ipcc662007.pdf $>$, [20 de noviembre de 2010].

LACOSTE, Y. Geografia del Subdesarrollo. Barcelona. Ariel. 1978. 336 p.

LAMO DE ESPINOSA, J. y URBANO TERRÓN, P. Repercusiones del cambio climático en la agricultura y la alimentación mundial. Madrid. FAO - España. 2007.

SANTAMARTA, J. Comer carne ¿es sostenible? [En línea]. 2007. http://www.kaosenlared.net/noticia/comer-carne-es-sostenible> [24 de noviembre de 2010].

SEGRELLES SERRANO, J. A. Problemas ambientales, agricultura y globalización en América Latina. [en línea]. Scripta Nova. Revista Electrónica de Geografía y Ciencias Sociales. vol. 5, no 92. 2001. 32 p. <http://www.ub.es/geocrit/sn-92.htm>, [21 de noviembre de 2010].

SEGRELLES SERRANO, J. A. La ecología y el desarrollo sostenible frente al capitalismo: una contradicción insuperable. Revista NERA. Año 11, n 12. 2008. 20 p. 
SEGRELLES SERRANO, J. A. Mundialización y espacio. Colloque Internacional: "Les societés de la mondialisation”. Nantes. 2004.

SEGRELLES SERRANO, J. A. La distribución agroalimentaria y su influencia en la pobreza campesina. [en línea]. Scripta Nova. Revista Electrónica de Geografía y Ciencias Sociales. vol. XIV, $\mathrm{n}^{\mathrm{o}}$ 325. 2010. 26 p. <http://www.ub.es/geocrit/sn$\underline{\text { 92.htm }}>$ [6 de diciembre de 2010].

SABERES Y SABORES. Desnutrición infantil en Argentina. [en línea] $<$ http://www.saberesysabores.com.ar/2006/saberes/mar/01006.htm $>\quad[27$ de diciembre de 2010].

STEINFELD, $\mathrm{H}$. El ganado emerge de la larga sombra. $18^{\circ}$ Congreso Mundial de la carne de OPIC. [en línea]. Buenos Aires. 2010. $<\underline{\text { http://www.congresomundialdelacarne2010.com/disertaciones.php }>}\left[\begin{array}{ll}6 & \text { de }\end{array}\right.$ diciembre de 2010].

VEGAN SOCIETY. Problemas medioambientales de la producción de carne. [en línea]<http://ecosofia.org/2006/10/problemas_medioambientales_de_la produccion de_carne.html $>$. [24 de noviembre de 2010].

VV. AA., El clima, cambios, peligros y perspectivas. Madrid. Editorial Popular, S.A. 2007. 